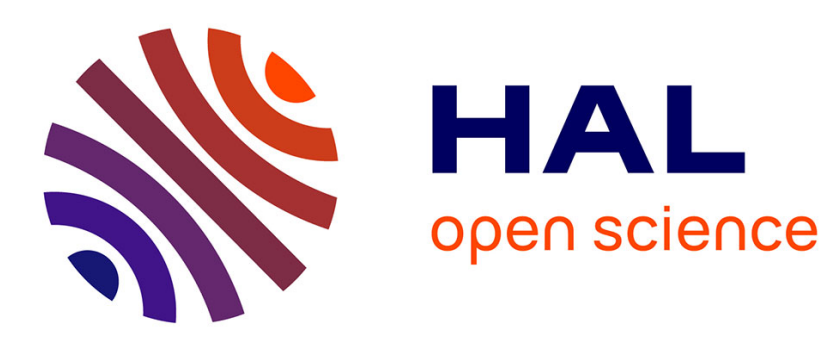

\title{
A Hybrid End-to-End QoS Path Computation Algorithm for PCE-Based Multi-Domain Networks
}

\author{
Ahmed Frikha, Samer Lahoud, Bernard Cousin
}

\section{To cite this version:}

Ahmed Frikha, Samer Lahoud, Bernard Cousin. A Hybrid End-to-End QoS Path Computation Algorithm for PCE-Based Multi-Domain Networks. Journal of Network and Systems Management, 2014, Journal of Network and Systems Management, 22, (4), pp.682-708. 10.1007/s10922-013-9273-5 . hal01134296

\section{HAL Id: hal-01134296 \\ https://hal.science/hal-01134296}

Submitted on 13 Apr 2015

HAL is a multi-disciplinary open access archive for the deposit and dissemination of scientific research documents, whether they are published or not. The documents may come from teaching and research institutions in France or abroad, or from public or private research centers.
L'archive ouverte pluridisciplinaire HAL, est destinée au dépôt et à la diffusion de documents scientifiques de niveau recherche, publiés ou non, émanant des établissements d'enseignement et de recherche français ou étrangers, des laboratoires publics ou privés. 


\title{
A Hybrid End-to-End QoS Path Computation Algorithm for PCE-based Multi-Domain Networks
}

\author{
Ahmed Frikha · Samer Lahoud . \\ Bernard Cousin
}

Received: date / Accepted: date

\begin{abstract}
the domains. domain or in multiple domains based on the PCE architecture.

Ahmed Frikha

University of Rennes 1, IRISA, 35042 Rennes Cedex, France

Tel.: +33.299842588

Web: www.ahmedfrikha.com

E-mail: ahmed.frikha@irisa.fr

Samer Lahoud

University of Rennes 1, IRISA, 35042 Rennes Cedex, France

E-mail: samer.lahoud@irisa.fr

Bernard Cousin

University of Rennes 1, IRISA, 35042 Rennes Cedex, France

E-mail: bernard.cousin@irisa.fr
\end{abstract}

Abstract The Inter-domain Quality of Service (QoS) routing is a challenging problem for today's Internet. This problem requires the computation of paths that cross multiple domains and meet the different QoS constraints. In addition, the methods of computation that are used must meet the constraints of confidentiality and autonomy imposed by the domains of different operators. The path computation element (PCE)-based architecture offers a promising solution for the inter-domain QoS routing. It ensures the computation of endto-end QoS paths while preserving the confidentiality and the autonomy of

In this paper, we propose a novel hybrid end-to-end QoS path computation algorithm, named HID-MCP, for PCE-based networks. HID-MCP is a hybrid algorithm that combines the advantages of pre-computation and ondemand computation to obtain end-to-end QoS paths. Moreover, it integrates crankback mechanisms for improving the path computation results in a single

The simulation results show that our algorithm has an acceptance rate of the requests very close to the optimal solution. Moreover HID-MCP outperforms BGP in terms of success rate and the difference is up to $30 \%$ in a realistic network. Detailed analysis are provided to assess the performance of our algorithm in terms of success rate and computational complexity. Besides, 
our solution relies on the PCE architecture to overcome the limitations related to inter-domain routing such as domain autonomy and confidentiality.

Keywords QoS routing · inter-domain routing · path computation element $(\mathrm{PCE}) \cdot$ crankback mechanisms $\cdot$ pre-computation $\cdot$ on-demand computation

\section{Introduction}

Nowadays, diverse advanced applications are provided over IP-based networks (e.g. IPTV, video-on-demand, and VoIP). Guaranteeing the Quality of Service (QoS) to such applications is a difficult problem, especially when service delivery requires crossing heterogeneous domains under the responsibility of different operators. In such a case, the problem becomes more complex. Moreover, operators adopt different policies which consolidate their economic interests and confidentiality clauses. Thus, cooperation between operators for providing QoS is possible only if it satisfies their policies. Routing is one of the primary mechanisms for providing QoS. It consists in the computation of an end-to-end path which ensures the delivery of the service while meeting the QoS constraints. Several recent works studied the multi-domain QoS routing. Yannuzzi et al [1] presented the challenges of finding disjoint QoS paths in multi-domain networks. One of the most challenging problems is the domain visibility. In fact, information about domain topology and QoS metrics are confidential and cannot be exchanged between domains. This makes finding an inter-domain QoS path a hard task. Some other studies proposed to aggregate QoS information and exchange them between domains. Uludag et al [2] provided an analysis of the different techniques of topology aggregation for QoS routing.

The path computation element (PCE)-based architecture [3] offers a promising solution for the inter-domain QoS routing. It enables computing end-to-end QoS paths in multi-domain networks while preserving confidentiality clauses and autonomy of the domains by distributing the computations over the domains. This architecture supposes that each domain has one or more PCE responsible of the intra-domain computation and can cooperate with other PCE using the PCE-to-PCE protocol in order to compute an end-to-end path [4]-[5]. The backward-recursive procedure (BRPC) is introduced by Vasseur et al [6] for the multi-domain path computation. This procedure relies on the PCE architecture to compute an end-to-end QoS path that meets the QoS requirement. This procedure allows PCE to exchange aggregated paths with other PCE using a compact structure, called virtual shortest path tree (VSPT). The drawback of this procedure is that it does not allow domains to exchange all aggregated paths but only one single path per entry border node. Geleji et al [7] provided a comparison of different schemes for the end-to-end path computation based on the PCE architecture.

In this paper, we propose a novel inter-domain QoS routing algorithm relying on the PCE-based architecture, named HID-MCP. HID-MCP is based on a hybrid computation scheme. The hybrid computation scheme combines 
the advantages of the pre-computation scheme [9]-[10] such as the low computational time and the advantages of the on-demand computation such as the high acceptance rate of the requests [11]. Our algorithm consists of two phases: An offline phase and an online phase. In the offline phase, HID-MCP pre-computes a set of QoS paths, as well as look-ahead information for each domain. Look-ahead information gives a measure of the best QoS performance that can be provided by the domain. In the online phase, HID-MCP combines the pre-computed paths to obtain an end-to-end path that fulfills the QoS constraints. Combining the pre-computed paths does not lead always to an end-to-end path. In such a case, a crankback mechanism is executed to perform on demand computations. Combining pre-computation and on-demand computation using a crankback mechanism improves the computation results and allows computational complexity to be reduced.

We distinguish two different crankback mechanisms: intra-domain crankback and inter-domain crankback. The intra-domain crankback is solicited to locally improve the computation results by executing an on-demand computation in the failing domain, while the inter-domain crankback performs a global improvement by executing an on-demand computation starting from one of the downstream domains in the crossed domain set. Note that this solution extends our recently published work in [8] by enhancing the algorithm side and simulation side. We enhance the algorithm by adding a new parameter, named hops. This parameter represents the number of backward hops in term of domain to be done before executing the on-demand computation. Precisely, in [8], the inter-domain crankback signaling is sent to the destination domain. While in this paper, the inter-domain crankback signaling can be sent to one of the downstream domains in order to execute the on-demand computation starting from this domain, and not only starting from the destination domain. This parameter allows us to tune the performance of the algorithm, and to ensure a trade-off between the rapidity and the success rate of the algorithm. We detail the operation performed by HID-MCP using some examples. We improve the simulation side by considering larger and realistic networks. We also compare the average computational time of the path combination and the on-demand computation in order to show the advantage of using pre-computation. Finally, we study the performance of our algorithm by comparing it with two algorithms. The first algorithm is an exact one, and it represents the optimal solution. The second algorithm is based on the Border Gateway Protocol (BGP), and it represents the inter-domain routing protocol employed in today's Internet. The simulation results show that our algorithm has an acceptance rate of the requests very close to the optimal solution. Moreover HID-MCP outperforms BGP in terms of success rate and the difference is up to $30 \%$ in a realistic network. Detailed analysis are provided to assess to the performance of our algorithm in terms of success rate and computational complexity. Besides, our solution relies on the PCE architecture to overcome the limitations related to inter-domain routing such as domain autonomy and confidentiality. 


\section{The Inter-Domain QoS Routing Problem}

Inter-domain QoS routing, also known as Inter-Domain Multi-Constraint Path (ID-MCP) computation problem, consists in computing a path subject to multiple QoS or cost constraints between a source and a destination node of a multi-domain network. Computing this path requires the knowledge of the QoS metrics on the network links. The QoS metrics can be classified into three types: bottleneck metrics such as bandwidth, additive metrics such as delay, and multiplicative metrics such as loss rate. The multiplicative metrics can be translated into additive metrics using the logarithm function. The bottleneck metrics can be resolved by omitting all links which violate the constraints and then computing the path on the residual graph. Therefore, we consider in the following, only additive metrics.

Let us introduce some notations to formally define the ID-MCP problem. Let $G(N, E, D)$ denote a graph of a multi-domain network, $N$ is the set of nodes, $E$ is the set of links and $D$ is the set of domains. The graph $G$ is composed of $D$ domains. Let $m$ be the number of QoS constraints. An $m$ dimensional weight vector is associated with each link $e \in E$. This vector consists of $m$ non-negative QoS weights $w_{i}(e), i=1 . . m$. Let $p$ be a path in the graph $G(N, E, D)$ and $w_{i}(p)$ be the weight of $p$ corresponding to the metric $i$. As metrics are additive, $w_{i}(p)$ is given by the sum of the weights of the $i^{t h}$ metric of the links of the path $p: w_{i}(p)=\sum_{e_{j} \in p}\left(w_{i}\left(e_{j}\right)\right)$. Let $\vec{W}(p)=$ $\left(w_{1}(p), w_{2}(p), \ldots, w_{m}(p)\right)$ denote the weight vector of the path $p$.

\section{Definition 1}

Given a source node $s$, a destination node $d$ and a set of constraints given by the constraint vector $\vec{C}=\left(c_{1}, c_{2}, . ., c_{m}\right)$, the Inter-Domain Multi-Constraint Path (ID-MCP) computation problem consists in finding a path $p$ which satisfies $w_{i}(p) \leq c_{i}, \forall i \in 1$..m. Such a path $p$ is called a feasible path.

The ID-MCP problem belongs to $\mathcal{N} \mathcal{P}$-Complete problem [12] and may have zero, one or multiple solutions (feasible paths). Beside, information about the internal topology or the QoS metrics on the links is confidential since the operators can be in competition. Therefore, computing such a path while meeting all of these constraints is a challenging task.

Currently, the universal inter-domain routing protocol is BGP. Although BGP has proved its efficiency to enable inter-domain routing with confidentiality and autonomy concern, this protocol cannot solve the ID-MCP problem since it does not take into account QoS constraints. One major limitation of BGP is the unique path propagation. BGP cannot propagate several paths for the same destination which provides from QoS-aware inter-domain routing. Another problem is that BGP performs the inter-domain route selection without any coordination between the domains. To overcome these limitations many extensions for BGP are proposed to support QoS routing [13]-[14]. However, the QoS capabilities of these propositions remain limited, due to the fact 
that only one path per destination can be propagated from a domain to another neighbor domain. Therefore, it is not possible to find an end-to-end path that meets the QoS constraints using these propositions.

The research community has recently been exploring the use of distributed architecture to solve the ID-MCP problem. The IETF defines in [3] an alternative architecture based on Path Computation Elements (PCE) that enable the cooperation between domains to find a path subject to multiple constraints. In this architecture, the domains designate one or more PCE, able to perform advanced routing computations within the domain, to cooperate with external PCE and then offer the inter-domain cooperation needed for QoS end-to-end routing. Moreover, the PCE-based architecture preserves confidentiality and the autonomy of each domain by distributing the computation over the domains. Each domain computes locally the segment of the end-to-end path that traverses it.

To our knowledge, few works have been proposed to solve the ID-MCP problem using the PCE architecture. The algorithm proposed in [15] extends the exact algorithm SAMCRA [16] to an inter-domain level to solve the IDMCP problem. The drawback of this algorithm is its high complexity. The IDPPPA algorithm [17] and the ID-MEFPA algorithm [18] rely on the PCE architecture and attempt to solve the ID-MCP algorithm using a pre-computation scheme. These solutions are efficient in terms of rapidity and have a good success rate. The pre-computation scheme ensures a very low computational time, but requires a periodical update of the network link state information to maintain a high success rate. Work in [19] proposes also a promising distributed solution with crankback mechanisms for inter-domain routing. However this solution cannot take into account several QoS metrics.

\section{Computation Schemes for Inter-domain QoS Routing}

Different computation schemes have been proposed in the literature to solve the QoS routing problem [11]. The on-demand computation scheme attempts to find a feasible path for each request using network state information. The computation is triggered upon the reception of a QoS request. This computation scheme provides a high probability of finding a feasible path since the network state information is up-to-date [11]. This scheme is widely used in existing networks but presents some serious limitations with the emerging applications in the Internet. In fact, the ID-MCP problem belongs to $\mathcal{N} \mathcal{P}$-Complete problem, consequently, the performance of on-demand routing in terms of response time is severely affected. In contrast with the on-demand computation scheme, the pre-computation scheme allows the QoS routing problem to be solved while speeding up the response time [9]-[10]. The pre-computation proceeds in two phases: It prepares in advance a set of paths satisfying predetermined QoS requests. Then, at the reception of a QoS request, it attempts to rapidly provide a feasible path among the pre-computed paths. However, the deployment of a pre-computation scheme in networks can run into problems. 
One problem is that the stored paths are computed based on a snapshot of the network state taken before the reception of the QoS request [11]. Consequently, the pre-computed paths do not necessarily satisfy the QoS requests after a change in the network state [11]. In addition, a pre-computation scheme cannot predict all possible QoS requests even if the snapshot remains valid. This decreases the success rate of the pre-computation scheme. For these reasons, a hybrid computation scheme is proposed. This scheme combines the two aforementioned computation schemes and is performed in two phases. As with the pre-computation scheme, the first phase consists in preparing in advance a set of paths or segment of paths. The second phase is more sophisticated. When the pre-computed paths do not lead to a path which satisfies the request, on-demand computation is triggered. Therefore, the hybrid computation scheme ensures a high acceptance rate for requests while reducing the computation time. In this paper, we rely on a hybrid computation scheme to solve the ID-MCP problem.

\section{The HID-MCP Algorithm}

In this paper, we propose a novel inter-domain QoS routing algorithm based on a hybrid computation scheme and named HID-MCP (Hybrid ID-MCP). The present section details the operations performed by this algorithm. Fig. 1 illustrates the building block architecture of the algorithm. This architecture is implemented in each PCE of each domain. Based on this architecture, each PCE performs autonomous computations and cooperates with other PCE to compute the end-to-end path. This reinforces domain autonomy and confidentiality. As shown in Fig. 1, the HID-MCP algorithm consists of two phases. In the first phase, named the offline path computation phase, the algorithm executes a path segment computation procedure and computes look-ahead information in each domain. The pre-computed path segments and look-ahead information are stored in a database for later use. The second phase, named online path computation phase, is triggered upon the reception of a QoS request. In this phase, HID-MCP computes an end-to-end path that spans multiple domains and fulfills the QoS constraints. The algorithm attempts to find such a path, first by combining the stored pre-computed intra-domain path segments, second by executing an on-demand path computation procedure that takes benefits from the stored look-ahead information to speed up the computational time of the algorithm.

\subsection{The Offline Path Computation Phase}

The offline computation phase consists of computing in advance a set of intradomain paths subject to multiple predetermined QoS constraints. It also computes look-ahead information at the level of each entry border node of the corresponding domain. In the following, we detail the operations involved in these two computations. 
Offline path computation phase

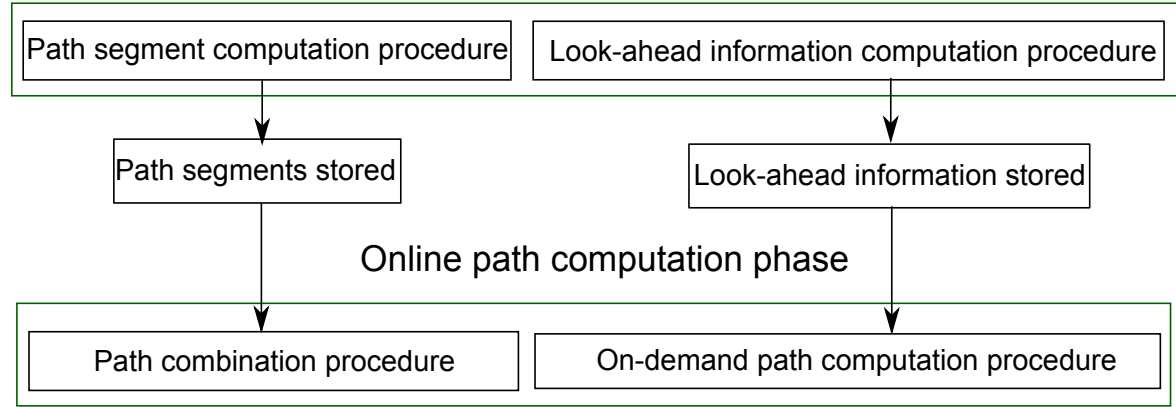

Fig. 1 The building block architecture of the HID-MCP algorithm.

\subsubsection{The Path Segment Computation Procedure}

This procedure pre-computes a set of paths from each entry border node of the domain toward the other nodes of this domain as well as the entry border nodes of the neighbor domains. These paths satisfy a set of predetermined additive QoS constraints. In practice, some QoS metrics are more critical for certain applications, such as the delay for the VoIP-based applications. Therefore, our procedure pre-computes for each single QoS metric the path which minimizes the weight corresponding to this metric. For example, it pre-computes the path which minimizes the delay; this path can be useful for the VoIP-based applications.

Let $D_{q}$ be the considered domain, $n_{1}$ be a border node of $D_{q}, n_{2}$ be a node of $D_{q}$ or an entry border node of a neighbor domain, and $m$ be the number of the QoS metrics, our procedure computes $m$ shortest paths from $n_{1}$ to $n_{2}$. Each shortest path minimizes a single QoS metric. Hence, from each entry border node $n_{1}$ of $D_{q}$, this procedure computes $m$ shortest path trees. Each shortest path tree is computed using the Dijkstra algorithm and considering a single metric. Therefore, our procedure executes Dijkstra $m$ times per border node. The complexity of the path segment computation procedure is given by equation (1), where $B$ is the number of the entry border nodes of the domain.

$$
\mathcal{O}(B * m(N \log (N)+E))
$$

The complexity of this procedure depends on the number of constraints $m$. For one border node, this procedure is in $\mathcal{O}(m(N \log (N)+E))$ corresponding to $m$ times the complexity of Dijkstra, which is $\mathcal{O}((N \log (N)+E))$. Considering the $B$ entry border nodes of the domain, the global complexity is then in: $\mathcal{O}(B * m(N \log (N)+E))$.

\subsubsection{Look-Ahead Information Computation Procedure}

During the offline phase of HID-MCP, we propose the computation of lookahead information in each domain. This information gives a measure of the 
best QoS performance that can be provided by the domain. Particularly, it allows the computation search space of a potential on-demand path computation procedure to be reduced. For instance, this information allows infeasible paths to be discarded from the search space of the procedure before exploring these paths. Therefore, look-ahead information reduces the computational complexity of the online phase and contributes to maintain a reasonable response time. Look-ahead information is inferred from the result of the pre-computation algorithm. Let $n_{1}$ be a border node of the domain, and $n_{2}$ be a node of the domain or an entry border node of a neighbor domain, and $p_{n_{1} \mapsto n_{2} ; i}^{*}$ denotes the pre-computed shortest path between node $n_{1}$ and node $n_{2}$ considering the metric $i$. The weight $w_{i}\left(p_{n_{1} \mapsto n_{2} ; i}^{*}\right)$ is the lowest possible path weight between $n_{1}$ and $n_{2}$. Similarly, let us denote by $\vec{W}^{*}{ }_{n_{1} \mapsto n_{2}}=\left(w_{1}^{*}, . ., w_{m}^{*}\right)$ the vector where $w_{i}^{*}=w_{i}\left(p_{n_{1} \mapsto n_{2} ; i}^{*}\right)$. Then, $\vec{W}^{*}{ }_{n_{1} \mapsto n_{2}}$ represents the lowest weights to reach $n_{2}$ from $n_{1}$ for each single metric. We note that a path does not necessarily exist with these lowest weights for all the metrics simultaneously. However, this vector can be used in the online path computation phase to discard infeasible paths from the search space.

The complexity of the look-ahead information computation procedure is given by equation (2).

$$
\mathcal{O}(m * N * B)
$$

Look-ahead information is inferred from the result of the path segment computation. At each entry border node of the domain, there are at most $m * N$ stored pre-computed paths. Hence, at the level of an entry border node $n$ the complexity of computing the $N$ vectors $\vec{W}^{*}{ }_{n \mapsto n_{j}}$, where $n_{j} \in N$, is in $\mathcal{O}(m * N)$. Therefore, the complexity of computing the look-ahead information for all the entry border nodes of the domain is in $\mathcal{O}(m * N * B)$.

\subsection{The Online Path Computation Phase}

The online path computation consists in finding a feasible end-to-end path using the pre-computed paths and taking advantage of the look-ahead information. Upon the reception of a QoS request, the source and the destination domains are determined. According to the cooperation policy, the service provider computes the best domain sequence that links the source and the destination domain using the PCE-to-PCE protocol [4]. Operators can also base their selection for the best domain sequence according to the number of traversed domains, i.e. by selecting the shortest domain sequence. Furthermore, selecting the best domain sequence will reduce the complexity of the problem by limiting the path computation in the domain sequence and not in all domains. The path computation is triggered in the destination domain toward the source domain following the selected domain sequence. Note that, without loss of generality, we rely on the backward-recursive procedure (BRPC), introduced by Vasseur et al [6], for the end-to-end path computation. 


\begin{tabular}{|l|l|}
\hline Algorithm 1 & \\
\hline$s$ & The source node \\
\hline$d$ & The destination node \\
\hline$S e q$ & The selected domain sequence $S e q=\left\{D_{1}, D_{2}, . ., D_{r}\right\}$ \\
\hline$D_{1}$ & The number of domains in $S e q$ \\
\hline$D_{r}$ & The destination domain \\
\hline$H_{q}$ & The source domain \\
\hline$h o p s$ & The VSPH received in the domain $D_{q}$ \\
\hline Algorithm 2 & The number of backward hops in terms of domains for the crankback \\
\hline$P_{q}$ & The set of pre-computed paths in domain $D_{q}$ \\
\hline$E_{q}$ & The set of egress nodes in domain $D_{q}$ \\
\hline$I_{q}$ & The set of ingress nodes in domain $D_{q}$ \\
\hline$m$ & The number of constraints \\
\hline Algorithm 3 & \\
\hline$l$ & The maximum number of shortest paths selected from a VSPH \\
\hline$k$ & The parameter of TAMCRA \\
\hline$\Psi_{q}$ & Set of look-ahead information in domain $D_{q}$ \\
\hline$L_{q}$ & Set of look-ahead information in domain $D_{q}$ from $I_{q}$ to $E_{q}$ \\
\hline
\end{tabular}

Table 1 The used notations in all the algorithms of the online computation

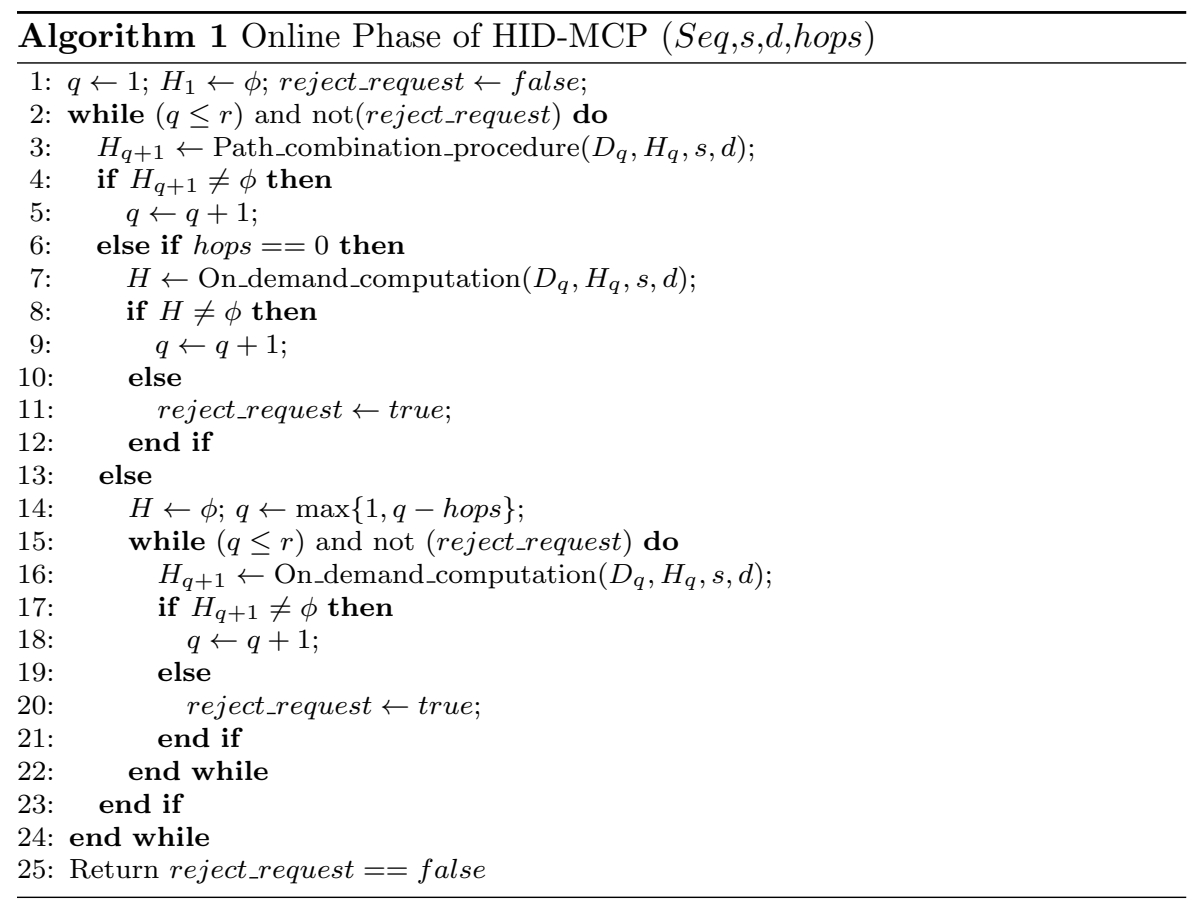

Table 1 illustrates the used notations in all the algorithms of the online computation. As shown in this table $S e q=\left\{D_{1}, D_{2}, . ., D_{r}\right\}$ denote the selected domain sequence, where $D_{1}$ is the destination domain and $D_{r}$ the source domain. $d$ is the destination node and $s$ is the source node. Algorithm 1 illustrates 
the operations performed in the online phase of HID-MCP. First, our algorithm attempts to compute an inter-domain path by combining the pre-computed paths in each domain $D_{q}$ in $S e q$ starting from the destination domain $D_{1}$ : the path combination procedure is called (line 3 ). Operations performed by this procedure are detailed in section 4.2.1. The result of the combination procedure in each domain $D_{q}$ is a set of sub-paths linking the destination node to the entry border nodes of the up-stream domain $D_{q+1}$. These sub-paths are sent to domain $D_{q+1}$ to combine them with the pre-computed segments in domain $D_{q+1}$. To preserve domain confidentiality, sub-paths are communicated between domain under a novel compact structure named VSPH (Virtual Shortest Path Hierarchy ${ }^{1}$ ). This structure contains only the end nodes of the paths (the destination node and the entry border nodes of the up-stream domain) as well as the weight vector of each path. The VSPH received in domain $D_{q}$ is denoted by $H_{q}$ in algorithm 1 . A virtual path $p_{d \rightarrow n}$ is represented in the VSPH by $\left[d, n, \vec{W}\left(p_{d \rightarrow n}\right)\right]$, where $d$ is the destination node, $n$ is an entry border node of the upstream domain $D_{q+1}$, and $\vec{W}\left(p_{d \rightarrow n}\right)$ is the weight vector of $p_{d \rightarrow n}$.

Combining the pre-computed paths in each domain can lead to an endto-end path, as detailed in section 4.2.1. However in some cases, no feasible path is found, i.e. the returned VSPH is empty. We introduce in the following two novel approaches using crankback mechanisms in order to overcome this limitation. The first approach executes an intra-domain crankback while the second approach executes an inter-domain crankback. Both of these approaches perform an on-demand path computation. The aim of the on-demand path computation procedure is to provide better results than the pre-computed ones. Operations performed by this procedure are detailed in section 4.2.2.

The parameter hops in the algorithm represents the number of backward hops in terms of domains to be done before starting the on-demand computation. When hops $=1$ the on-demand computation should start from the downstream domain $D_{q-1}$, and when hops $=q-1$ the on-demand compuation starts from the destination domain $D_{1}$, etc. For the intra-domain crankback approach hops $=0$. The intra-domain crankback approach (lines 6-12) executes the on-demand path computation procedure in the current domain $D_{q}$, i.e. where the combination has failed. Then, if a feasible path is found in the current domain, this path is sent to the up-stream domain which will resume the path combination procedure. Otherwise, if the algorithm does not find a solution in the current domain, i.e. $H_{q+1}=\phi$, the request is rejected.

The inter-domain crankback approach (lines 13-23) executes the on-demand path computation procedure starting from the domain $D_{\max \{1, q-h o p s\}}$. When $q-$ hops $<1$, the computation starts from the destination domain $D_{1}$. Each domain executes the on-demand path computation procedure and sends the computed VSPH to the up-stream domain. The computation stops when an end-to-end path is found or when the on-demand path computation procedure

\footnotetext{
1 The hierarchy is a structure which enables the storage of multiple paths between any two nodes [20].
} 
does not find a solution, i.e. the returned VSPH is empty. In the latter case, the request is rejected.

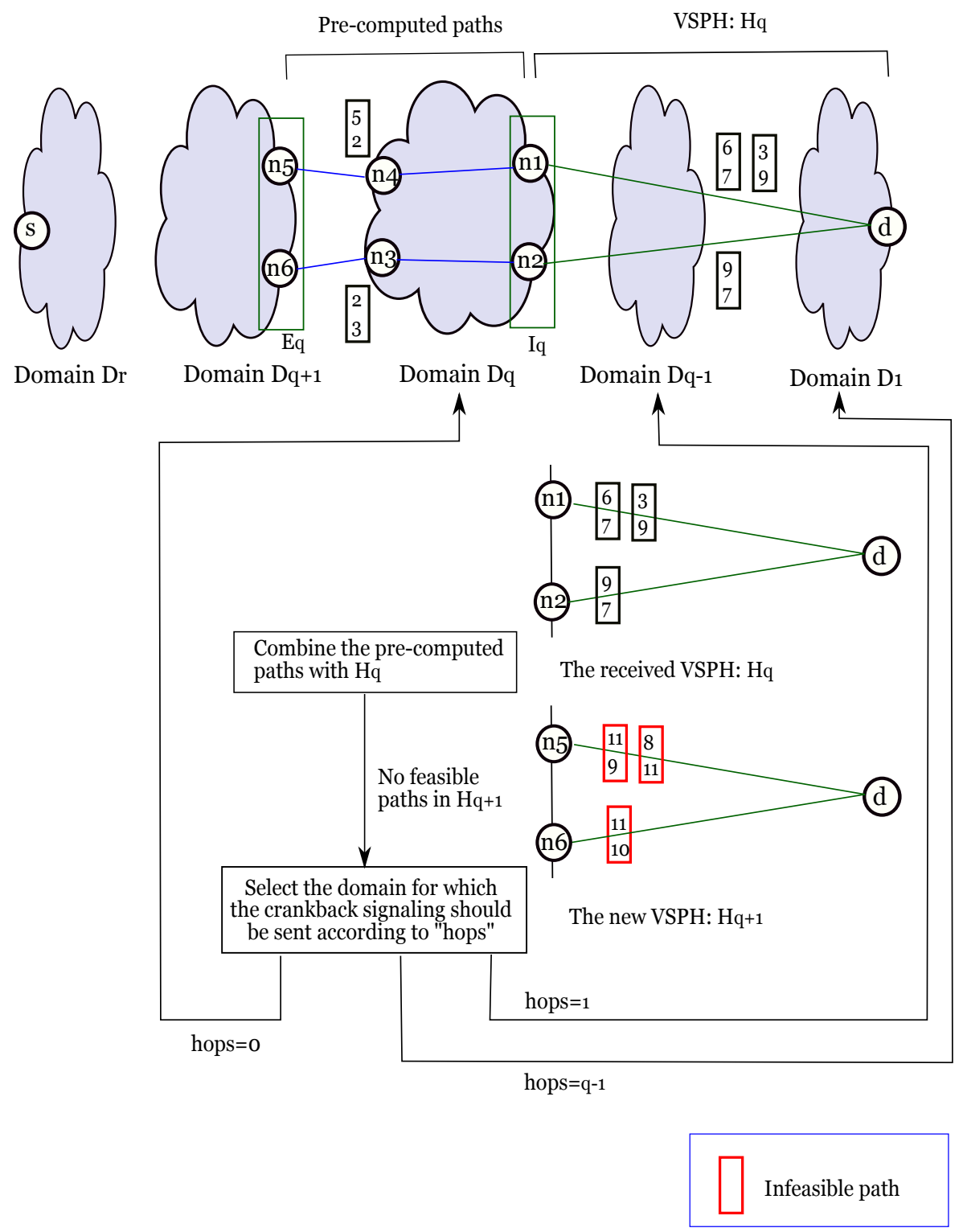

Fig. 2 Crankback signaling when the combination fails in domain $D_{q}$

Fig. 2 illustrates the crankback signaling when the combination fails in the domain $D_{q}$. In this example, the number of constraints $m=2$, and the constraint vector equals $(10,10)$. Domain $D_{q}$ receives the VSPH $H_{q}$ from domain 
$D_{q-1}$. It combines the pre-computed paths in $D_{q}$, with the aggregated paths in $H_{q}$. The combination procedure is detailled in the next section with an example. The result of the combination procedure is the VSPH $H_{q+1}$. However, $H_{q+1}$ does not contain any feasible paths. In this case, the crankback mechanism must be executed. According to the value of hops, the on-demand computation is executed in either the current domain $D_{q}($ hops $=0)$ or starting from a downstream domain in $\left\{D_{q-1}, D_{q-2}, . ., D_{1}\right\}$ (hops $>0$ ).

\subsubsection{Path Combination Procedure}

The aim of this procedure is to combine the paths in the received VSPH with the internally pre-computed one. Algorithm 2 illustrates the operations performed by the path combination procedure. First, the combination procedure selects the pre-computed paths linking nodes in the set $I_{q}$ to nodes in the set $E_{q}$, where $I_{q}$ is the ingress node set and $E_{q}$ the egress node set (lines 1-13). Then, these paths are combined with the aggregated paths received in the VSPH (line 17). Finally, feasible paths are aggregated and added to the new VSPH which will be sent to the upstream domain. Note that, at the level of the destination domain $D_{1}$ there is no received VSPH $\left(H_{1}=\phi\right)$, the procedure selects the feasible pre-computed paths linking the destination $d$ to the entry border nodes of domain $D_{2}$, and aggregates them in a VSPH to be sent to domain $D_{2}$.

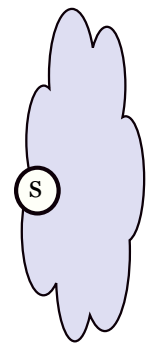

Domain Dr
Pre-computed paths

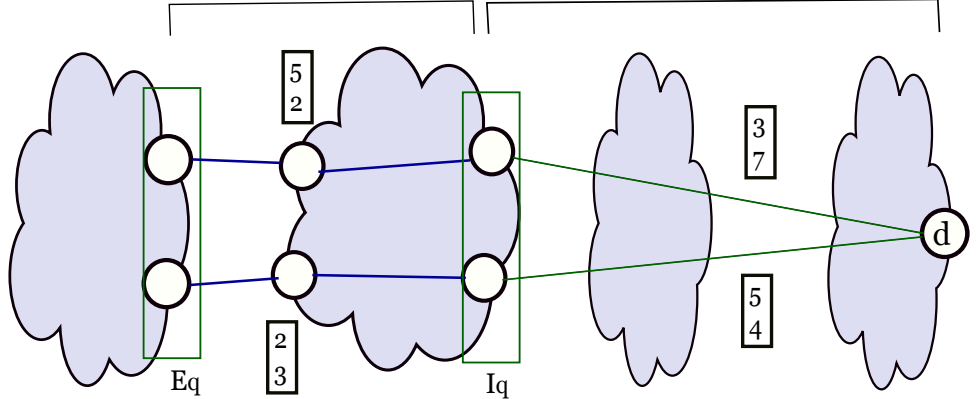

Domain $\mathrm{Dq}+1$
Domain Dq
VSPH: Hq

Domain D1

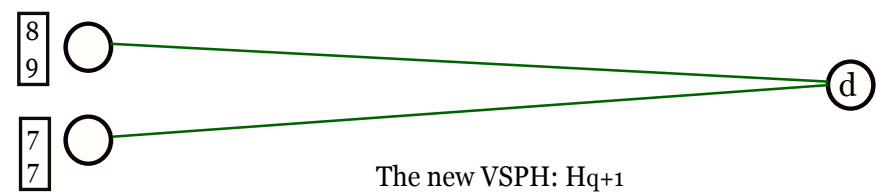

Fig. 3 Combining the pre-computed paths in domain $D_{q}$ with the VSPH.

Fig. 3 illustrates an example of path combination with two constraints $(m=2)$ in an intermediate domain $D_{q}$. In this example the constraint vector 


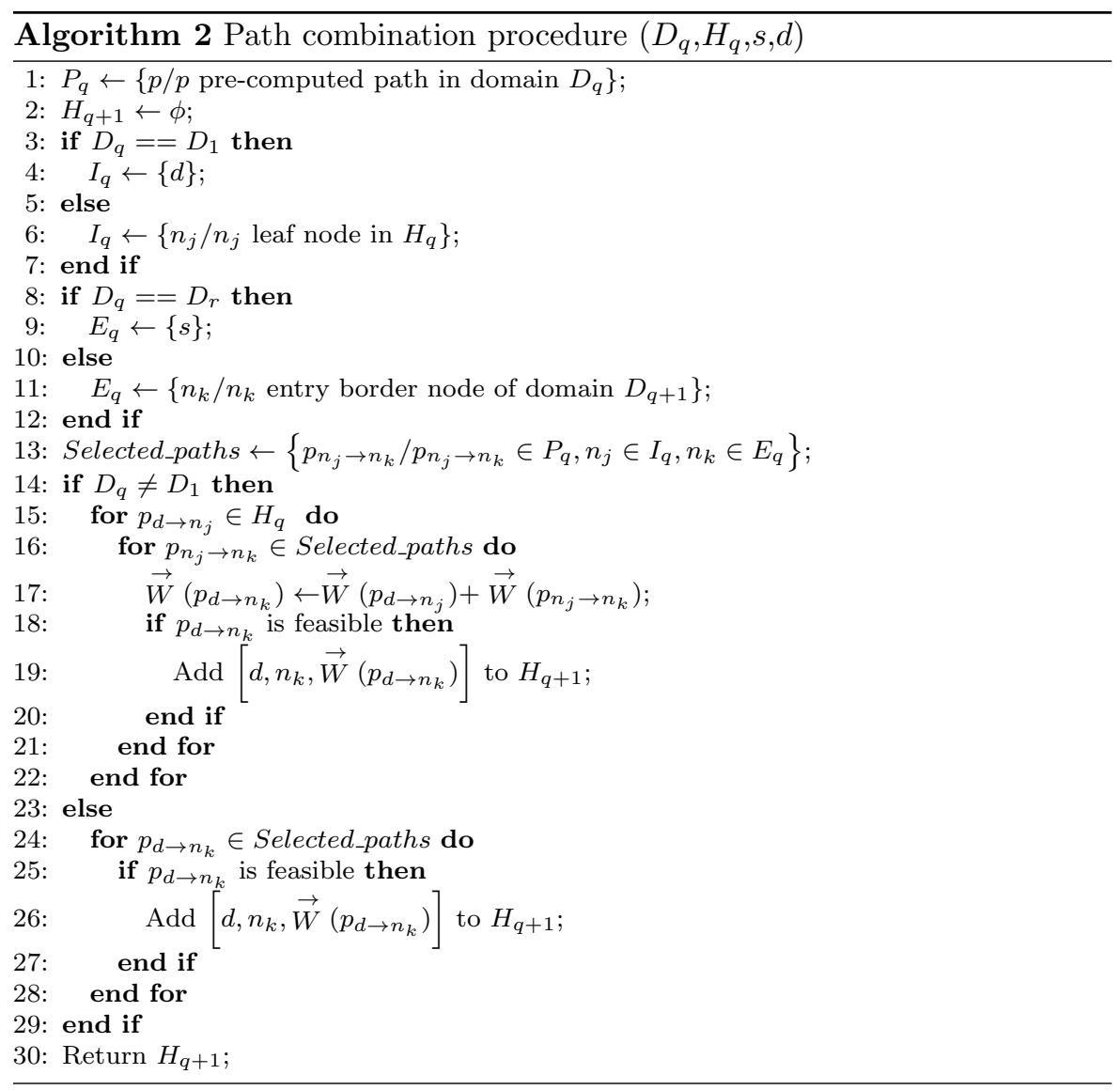

equals $\vec{C}=(10,10)$. Domain $D_{q}$ pre-computes two paths corresponding to the weight vectors $(5,2)$ and $(2,3)$. The VSPH $H_{q}$ contains two aggregated paths from the destination $d$ to the domain $D_{q}$. The weight vectors of these aggregated paths are respectively $(3,7)$ and $(5,4)$. We combine the aggregated paths with the pre-computed ones and we obtain two paths corresponding to the weight vectors $(8,9)$ and $(7,7)$. These two paths are aggregated in a new VSPH $H_{q+1}$ and then sent to domain $D_{q+1}$.

The complexity of the pre-computed path combination procedure at the level of an intermediate domain $D_{q} \in\left\{D_{2}, . ., D_{r-1}\right\}$ is given by equation (3), where $B_{\max }$ denotes the maximum number of border nodes between two domains.

$$
\mathcal{O}\left(m^{q} * B_{\max }^{2}\right)
$$

There are at most $m^{q-1}$ paths from the destination to each entry border node of the domain $D_{q}$. In addition, at each entry border node, there are at most $m * B_{\max }$ stored pre-computed paths to reach the upstream domain $D_{q+1}$. Hence, the complexity of combining the pre-computed paths and the 
received paths at the level of an entry border node is in $\mathcal{O}\left(m^{q} * B_{\max }\right)$. This operation is performed at each entry border node between the domain $D_{q}$ and the downstream domain $D_{q-1}$. Therefore, the global complexity of this procedure at each domain is in $\mathcal{O}\left(m^{q} * B_{\max }^{2}\right)$.

\subsubsection{The On-demand Path Computation Procedure}

When the pre-computed path combination procedure does not lead to a feasible path, the on-demand path computation procedure is called in the current domain or starting from the domain $D_{\max \{1, q-h o p s\}}$ according to the two aforementioned approaches. We propose a modified version of the TAMCRA algorithm to perform the on-demand computation. Work in [21] shows that TAMCRA is an efficient tunable heuristic for the MCP problem. TAMCRA introduces a new parameter $k$ that limits the maximum number of stored paths at each intermediate node when searching for a feasible path. This parameter allows TAMCRA's performance to be tuned: the success rate can be improved by increasing $k$ at the expense of increased computational complexity.

Algorithm 3 illustrates the operations performed by the on-demand path computation procedure. First of all, our proposed procedure computes a prediction for the lowest weight vector to reach domain $D_{q+1}$ through each path in the received VSPH (lines 11-19). We define for each aggregated path $\left[d, n, \vec{W}\left(p_{d \rightarrow n}\right)\right]$ in the VSPH and for each node $n_{k}$ in $E_{q}$, a weight vector $\vec{W}^{*}\left(d \mapsto n_{j} \mapsto n_{k}\right)$ that represents the sum of the weight vector of the computed segment $p_{d \mapsto n_{j}}$ and the lowest weight vector to reach $n_{k}$ from $n_{j}$ (line 13). Therefore, $\vec{W}^{*}\left(d \mapsto n_{j} \mapsto n_{k}\right)=\vec{W}\left(p_{d \mapsto n_{j}}\right)+\vec{W}^{*}{ }_{n_{j} \mapsto n_{k}}$, where $\vec{W}^{*}{ }_{n_{j} \mapsto n_{k}}$ is given by the look-ahead information. Note that the weight vector $\vec{W}^{*}\left(d \mapsto n_{j} \mapsto n_{k}\right)$ is not necessarily associated to an existing path. Next, we discard infeasible paths from the VSPH. For that, we define a new score for each path $p$ given by: $S\left(p_{d \mapsto n_{j}}\right)=\min _{n_{k} \in E}\left\{\max _{i \in 1 . . m}\left(\frac{w_{i}^{*}\left(d \mapsto n_{j} \mapsto n_{k}\right)}{c_{i}}\right)\right\}$. This score represents the lowest score to reach $d$ through $p_{d \mapsto n_{j}}$. A path $p$, that has a score $S(p)>1$, is infeasible since it cannot lead to any node in $E_{q}$ while meeting the QoS constraints. Then, we classify the remaining paths in VSPH according to the score $S$. We select the $l$ shortest paths having the $l$ lowest scores, where $l$ is a parameter of HID-MCP. The parameter $l$ of HID-MCP is very important to reduce the computational complexity of the on-demand computation procedure and to decrease the number of paths exchanged between domains. After that, for each selected shortest path $p_{d \mapsto n_{j}}$, we initialize the node $n_{j}$ by the corresponding weight vectors $\vec{W}\left(p_{d \mapsto n_{j}}\right)$ and we execute the TAMCRA algorithm starting from node $n_{j}$ to reach the nodes in $E_{q}$. The parameter $l$ represents the maximum number of executing the TAMCRA algorithm. We note that at the destination domain, i.e. where the computations start, there is no received VSPH. Hence, the on-demand procedure executes 


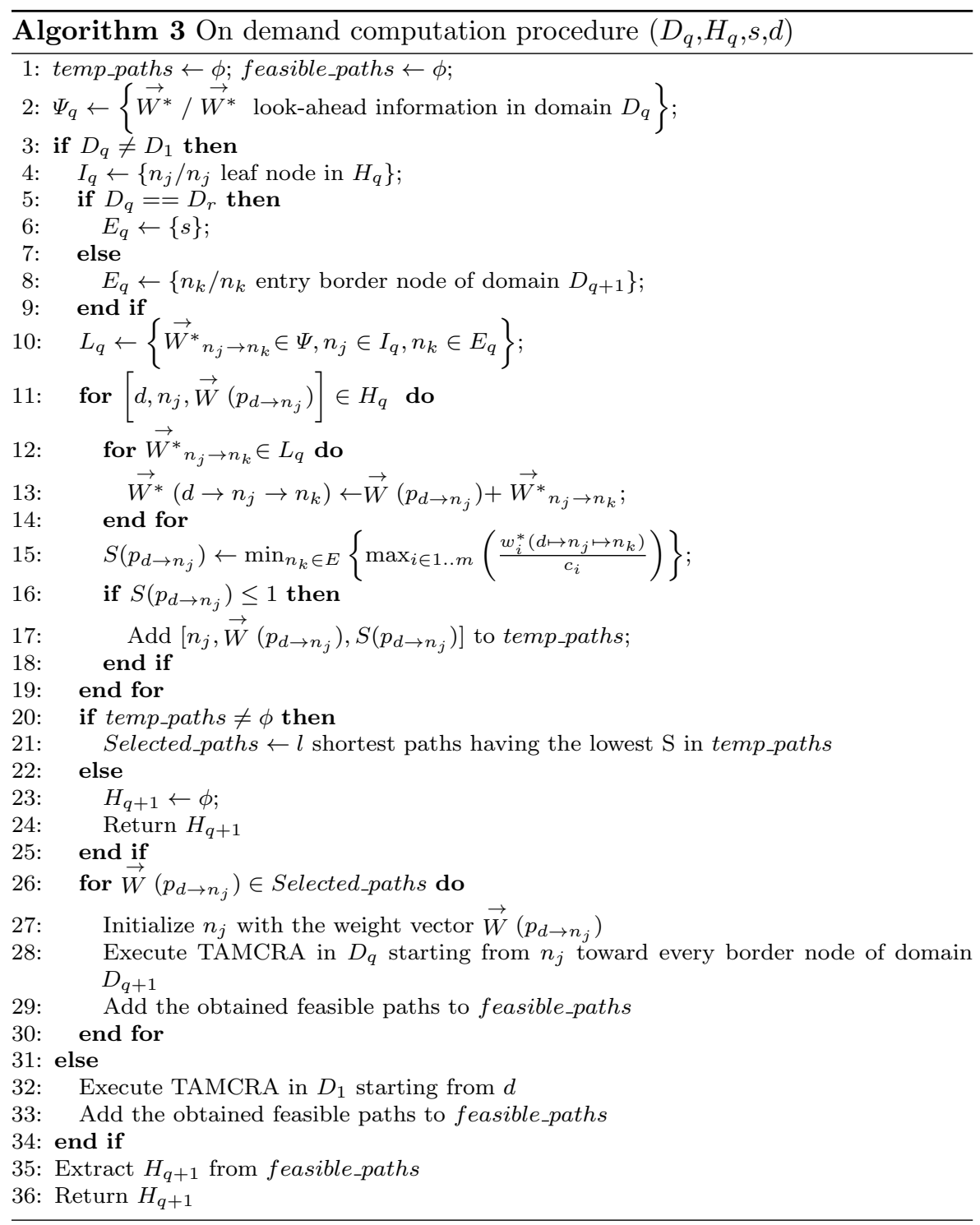

TAMCRA starting from the destination node. Finally, we aggregate the feasible paths computed by TAMCRA in a new VSPH.

Fig. 4 illustrates an example of on-demand computation in the intermediate domain $D_{q}$ with $m=2, k=2$ and $l=2$. In this example the constraint vector equals $\vec{C}=(10,10)$. The VSPH $H_{q}$ contains four aggregated paths from the destination $d$ to $D_{q}$. Two aggregated paths from $d$ to $n_{1}$ corresponding to the weight vectors $(5,3)$ and $(4,4)$. And two aggregated paths from $d$ to $n_{2}$ with the weight vectors $(5,3)$ and $(3,5)$. We combine the aggregated paths with 


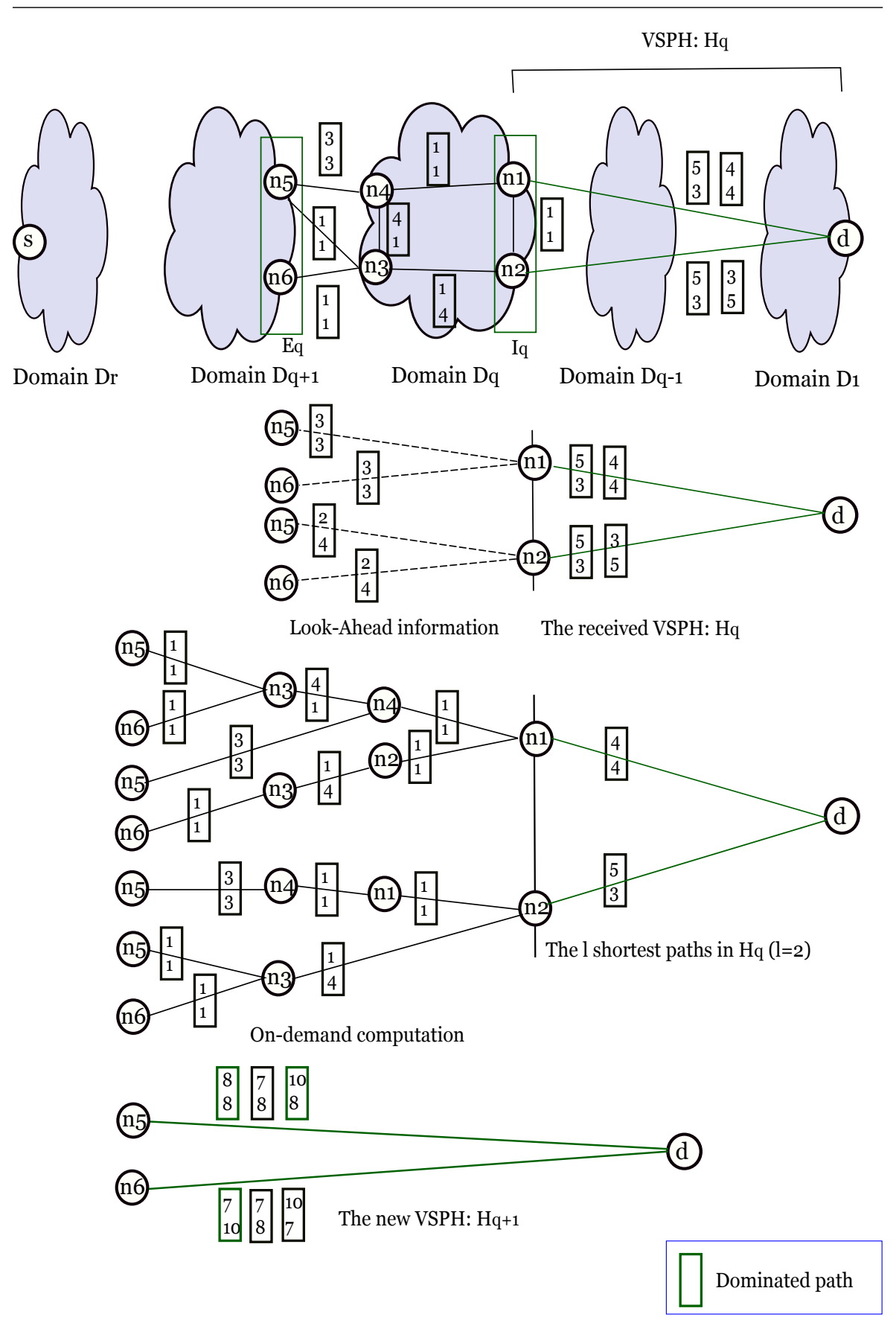

Fig. 4 The on-demand computation procedure in domain $D_{q}$ with $m=2, k=2$ and $l=2$ 
the look-ahead information in order to discard infeasible paths from $H_{q}$ and select the $l$ shortest paths from $H_{q}$. The two shortest aggregated paths in $H_{q}$ are $p_{d \mapsto n_{1}}$ and $p_{d \mapsto n_{2}}$ corresponding to the weight vectors $(4,4)$ and $(5,3)$, respectively. Finally, we initialize node $n_{1}$ with $(4,4)$ and node $n_{2}$ with $(5,3)$, and we execute the TAMCRA algorithm twice. One time starting form $n_{1}$ and the another time starting from $n_{2}$. We obtain three feasible paths from $d$ to $n_{5}$ with the weight vectors: $(8,8),(7,8)$ and $(10,8)$. $(8,8)$ and $(10,8)$ are dominated by $(7,8)$, so we discard them from the VSPH $H_{q+1}$. We also obtain three feasible paths from $d$ to $n_{6}$ with the weight vectors: $(7,10),(7,8)$ and $(10,7) .(7,10)$ is dominated by $(7,8)$, so we discard it from $H_{q+1}$.

The complexity of the on-demand path computation procedure at the level of an intermediate domain is given by equation (4).

$$
\mathcal{O}\left(l\left(k * N \log (k * N)+k^{2} * m * E\right)\right)
$$

The most significant point that determines the complexity of the on-demand path computation procedure is the number of executions of the TAMCRA algorithm. Knowing that the number of initialized node is less or equal to $l$, the complexity of this operation is in $\mathcal{O}\left(l\left(k * N \log (k * N)+k^{2} m * E\right)\right)$, corresponding to $l$ times the complexity of TAMCRA.

\section{Simulation and analysis}

In this section, we evaluate the performance of our algorithm HID-MCP in two steps. First, we perform an experimental study of the complexity of the two proposed approaches of HID-MCP: HID-MCP with intra-domain crankback mechanism, and HID-MCP with inter-domain crankback mechanism. We assess the performance of HID-MCP in terms of computational time, and percentage of requests requiring either an intra-domain or an inter-domain crankback mechanism. Second, we compare HID-MCP with some existing well-known inter-domain QoS routing algorithms. We run simulations using two different topologies:

- The first topology is a network of five domains. Each domain is built based on Waxman's model with 50 nodes in each one. The probability that two nodes of the network are connected by an edge is expressed in [22].

- The second topology has a symmetrical backbone and is called SYMCORE. SYM-CORE contains five interconnected domains and is taken from the work in [23]. This topology is used to assess the algorithms in a realistic case.

For these two topologies, we associate with each link two additive weights generated independently following a uniform distribution [10, 1023]. The QoS constraints are randomly generated according to the following: Let $p_{1}$ and $p_{2}$ denote the two shortest paths which minimize the first and the second metrics, respectively. Let $Z=\left[w_{1}\left(p_{1}\right), w_{1}\left(p_{2}\right)\right] \times\left[w_{2}\left(p_{2}\right), w_{2}\left(p_{1}\right)\right]$ be the constraint generation space. The problem does not belong to $\mathcal{N} \mathcal{P}$-Complete problem outside 
$Z$, i.e. either infeasible or trivial. As shown in figure 5 , we divide this space into ten zones $Z_{i}, i=1 . .10$ and we browse the space from the strictest constraint zone $Z_{1}$ to the loosest constraint zone $Z_{10}$. Then, we assess the performance of the algorithms according to these zones. In the following, each figure measures the variation of a performance metric according to the constraint generation zones $Z_{i}, i \in 1 . .10$, with a $95 \%$ confidence interval.

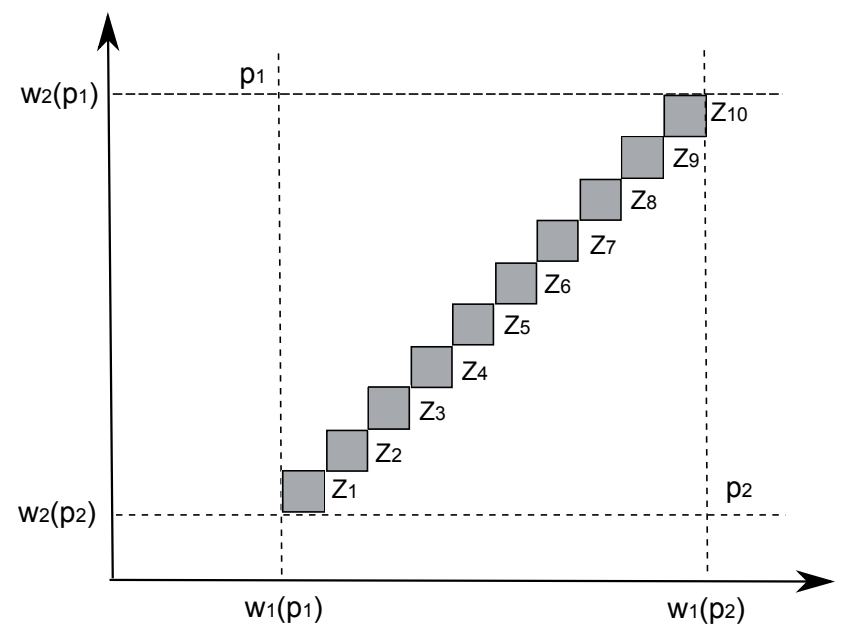

Fig. 5 Constraint generation zones for $m=2$

\subsection{Performance evaluation of HID-MCP}

Firstly, we compare the average computational time of the on-demand computation procedure and the combination procedure. This comparison will show us the importance of the pre-computation phase in reducing the computational time. In Table 2, we see the average computational time of the combination procedure and the on-demand computation procedure per domain according to the constraint generation zones $Z_{3}, Z_{5}, Z_{8}$ and $Z_{10}$, in the SYM-CORE topology. The combination procedure has a compuational time lower than $2 \times 10^{-3}$ seconds, while the on-demand compuation has a computational time higher than $1.8 \times 10^{-1}$ seconds.

\begin{tabular}{|c|c|c|c|c|}
\hline Constraint zones & $Z_{3}$ & $Z_{5}$ & $Z_{8}$ & $Z_{10}$ \\
\hline Combination Procedure & $4.26 \times 10^{-3} \mathrm{~s}$ & $4.37 \times 10^{-3} \mathrm{~s}$ & $4.7 \times 10^{-3} \mathrm{~s}$ & $5.81 \times 10^{-3} \mathrm{~s}$ \\
\hline On-demand Procedure & $1.73 \times 10^{-1} \mathrm{~s}$ & $2.09 \times 10^{-1} \mathrm{~s}$ & $2.34 \times 10^{-1} \mathrm{~s}$ & $2.97 \times 10^{-1} \mathrm{~s}$ \\
\hline
\end{tabular}

Table 2 Comparison of the average computational time of combination procedure and the on-demand computation procedure in the SYM-CORE topology. 
For the Waxman's model-based topology, Table 3 illustrates the average computational time of the combination procedure and the on-demand computation procedure in the constraint generation zones: $Z_{3}, Z_{5}, Z_{8}$ and $Z_{10}$. We see that the average computation time of the combination procedure is very low (almost lower than $2.6 \times 10^{-3}$ seconds) compared with that of the on-demand computation procedure (almost around 1.3 seconds).

\begin{tabular}{|c|c|c|c|c|}
\hline Constraint zones & $Z_{3}$ & $Z_{5}$ & $Z_{8}$ & $Z_{10}$ \\
\hline Combination Procedure & $2.43 \times 10^{-3} \mathrm{~s}$ & $2.6 \times 10^{-3} \mathrm{~s}$ & $2.55 \times 10^{-3} \mathrm{~s}$ & $2.52 \times 10^{-3} \mathrm{~s}$ \\
\hline On-demand Procedure & $1.27 \mathrm{~s}$ & $1.33 \mathrm{~s}$ & $1.33 \mathrm{~s}$ & $1.17 \mathrm{~s}$ \\
\hline
\end{tabular}

Table 3 Comparison of the average computational time of combination procedure and the on-demand computation procedure in the Waxman's model-based topology.

It is clear that the combination procedure is very fast compared with the on-demand computation. This is thanks to the pre-computation phase which computes in adavance multiple QoS paths and reduces considerably the compuational time of algorithm.

Secondly, we evaluate the success rate of the combination procedure and the need to execute either an intra-domain crankback or an inter-domain crankback in each domain. This study can help operators to take the best decision to execute either an intra-domain crankback or an inter-domain crankback, whenever the combination procedure fails to find an end-to-end feasible path. It also allows us to deduce the percentage of executions of the on-demand computation procedure in each domain for intra-domain crankback based HIDMCP. To determine if the intra-domain crankback may lead to a feasible path or not, we use the look-ahead information stored in the domain where the combination procedure has failed. Precisely, we combine the received VSPH with the look-ahead information, if all the obtained paths are infeasible, the intra-domain crankback cannot lead to a feasible path, and in such a case the inter-domain crankback is required. Otherwise, an intra-domain crankback may lead to a feasible path in the failed domain.

We first consider the Waxman's model-based topology. In this topology, the domain sequence is composed of five domains. The combination procedure is always successful in the three first domains of the domain sequence: the destination domain (i.e. $\left.D_{1}\right), D_{2}$ and $D_{3}$. The success rate of the combination procedure equals $100 \%$. The percentage of requests requiring either inter-domain crankback or intra-domain crankback equals zero. Therefore, the crankback mechanisms are never called from these domains.

Fig. 6, and Fig. 7 illustrate these percentages respectively in the intermediate domain $D_{4}$ and in the source domain $D_{5}$. The success rate of the combination procedure in $D_{4}$ is still high especially when the QoS constraints are not very strict (from $Z_{3}$ to $Z_{10}$ ). However, when constraints are strict, the combination can fail. For instance, in $D_{4}$ the success rate of the combination equals $38 \%$ in $Z_{1}$. The intra-domain crankback mechanism can lead to a fea- 


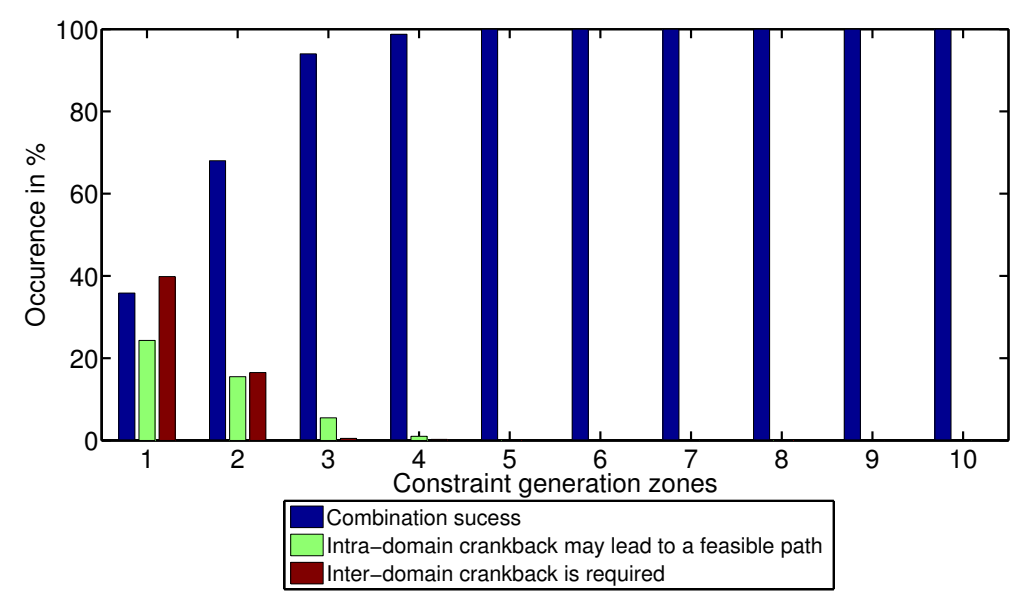

Fig. 6 Domain $D_{4}$ : Success rate of the combination procedure, percentage of requests requiring intra-domain crankback, and percentage of requests requiring the inter-domain crankback.

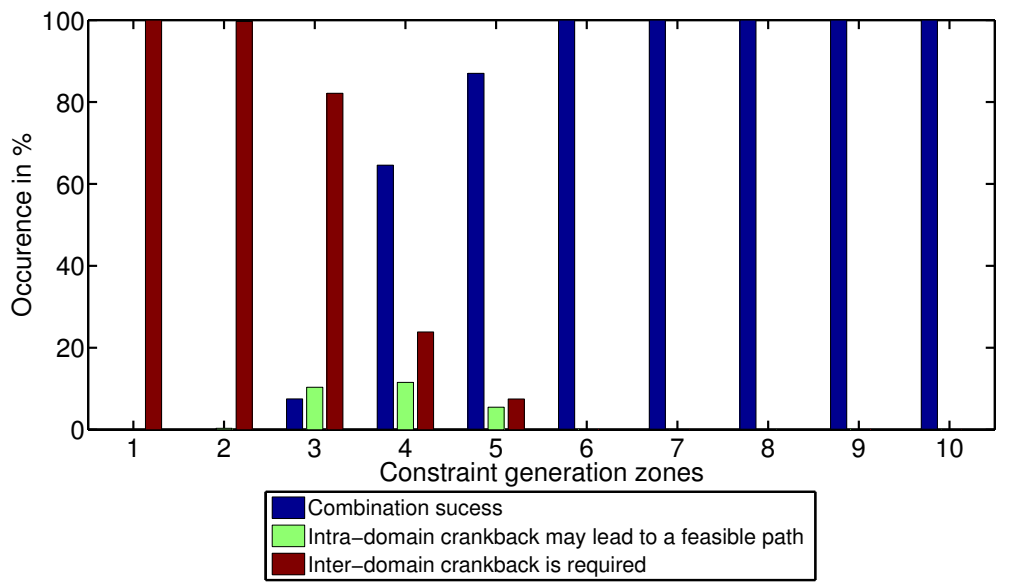

Fig. 7 Domain $D_{5}$ : Success rate of the combination procedure, percentage of requests requiring intra-domain crankback, and percentage of requests requiring the inter-domain crankback.

sible path in this domain and in $Z_{1}$ with a percentage that equals $40 \%$. For the remaining $22 \%$ of the cases, the inter-domain crankback is required.

In the source domain, we note that the success rate of the combination is low when the constraints are strict. Nonetheless, this procedure performs well when the constraints are less strict. In this domain, the percentage of requests requiring the inter-domain crankback equals $100 \%$ in $Z_{1}$ and $Z_{2}$. While, from $Z_{4}$ to $Z_{10}$ more than $77 \%$ of the requests do not require crankback mechanisms.

Now, we consider the SYM-CORE topology. We note that this topology is composed of five domains. The length of the domain sequence is three domains 


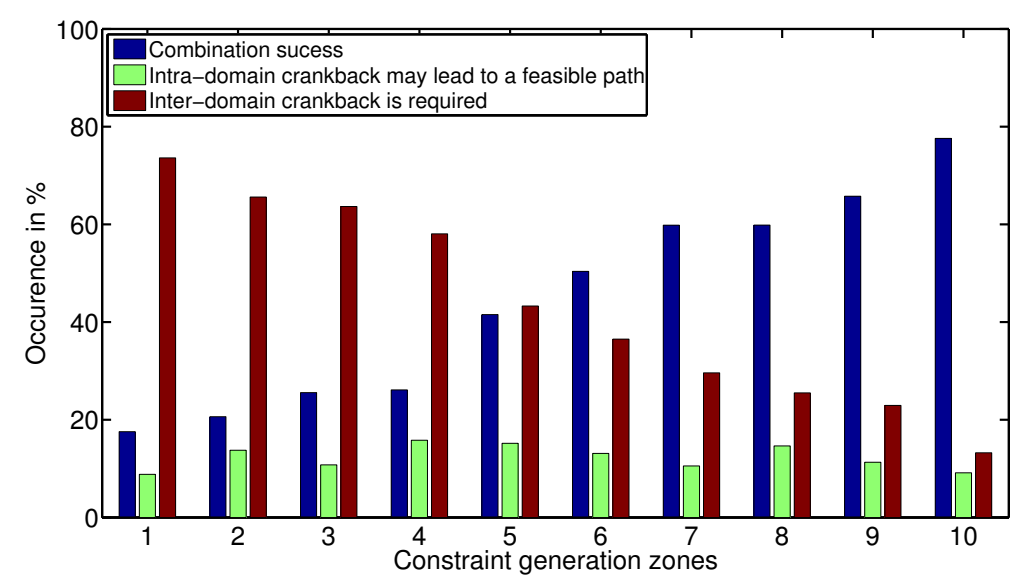

Fig. 8 Domain $D_{3}$ : Success rate of the combination procedure, and percentage of requests requiring intra-domain, and percentage of requests requiring the inter-domain crankback.

as SYM-CORE has a symmetrical backbone (i.e. all the domains are interconnected through one central domain). In the destination and the intermediate domains of the domain sequence, the percentage of success of the combination procedure equals $100 \%$ in all constraint generation zones. For the source domain, Fig. 8 shows the percentage of success of this procedure as well as the percentage of requests requiring the inter-domain crankback, and percentage of requests for which the intra-domain crankback may lead to a feasible path. The success rate of combination is good and increases when constaints are less strict. The inter-domain crankbak is required with a percentage lower than $43 \%$ from zone $Z_{5}$ to $Z_{10}$. All of these presented results prove that the global empirical complexity of HID-MCP remains reasonable especially when the constraints are not very strict, as the inter-domain crankback is required rarely in these zones.

Finally, we focus on the importance of the look-ahead information to discard infeasible paths before executing the on-demand computation procedure. We define the percentage of infeasible paths $(P I P)$ as the number of infeasible paths in the VSPH received by a specific domain over the total number of paths in this VSPH. Fig. 9 illustrates the percentage of infeasible paths $(P I P)$ in the VSPH received respectively, in the source domain (i.e. $D_{5}$ ), and in the intermediate domains (i.e. $D_{2}, D_{3}, D_{4}$ ). For the destination domain (i.e. $D_{1}$ ), there is no received VSPH as the computation starts from this domain. In Fig. 9, we see that the $(P I P)$ equals zero in $D_{2}$ and is lower than five percent in $D_{3}$. In fact, the weights of the paths in $D_{2}$ and $D_{3}$ are still low compared to the constraint vector. Therefore, detecting infeasible paths using the look-ahead information is unlikely to happen. However, in the source domain (i.e. $D_{5}$, the last domain in the computation process) the percentage of the discarded infeasible paths is very high, and equals $100 \%$ in the constraint generation 


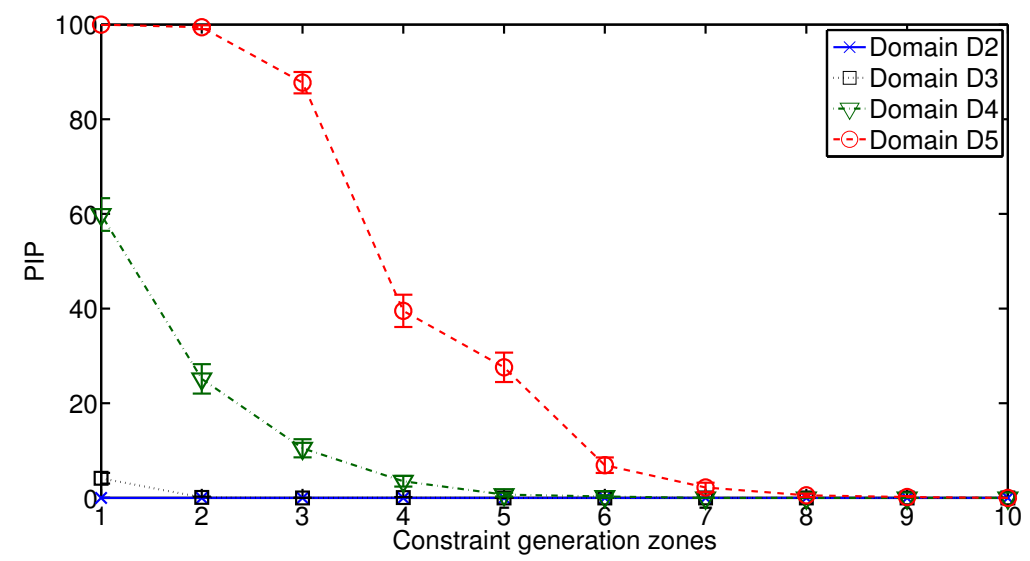

Fig. 9 Percentage of infeasible paths in the received VSPH in the Waxman's model-based topology.

zones $Z_{1}$ and $Z_{2}$. This means that no path in the received VSPH can lead to a feasible path. Therefore, in $D_{5}$ when the constraints are generated in $Z_{1}$ or $Z_{2}$, there is no need to execute the on-demand computation locally because it cannot find a feasible path. In this case operators have the choice between executing an inter-domain crankback or rejecting the request, depending on their policies and on the importance of request (e.g. priority of the request). In $D_{4}$, the PIP is high and equals $60 \%$ in the constraint generation zone $Z_{1}$. Thus, $60 \%$ of the paths will be discarded from the VSPH, and this will reduce the computational time of the on-demand computation procedure. These results prove the importance of look-ahead information in discarding infeasible paths, especially in $D_{4}$ and $D_{5}$. Nonetheless, the look-ahead information remains important in all crossed domains in order to select the $l$ shortest paths. As explained previously, the parameter $l$ is required to limit the number of executions of the TAMCRA algorithm when calling the on-demand computation procedure. Therefore, the lower is $l$ the faster is the on-demand computation.

\subsection{Comparaison of HID-MCP with existing approaches}

In this section, we evaluate the performance of the HID-MCP algorithm by comparing it with two different kinds of inter-domain routing algorithms: practical algorithms employed in today's Internet and exact QoS algorithms based on the PCE architecture. For the first type of algorithms, we select the Border Gateway Protocol (BGP), as it is the current inter-domain routing protocol employed in the Internet. This protocol has a low computational complexity. However, it does not allow domains to exchange information about the QoS metrics. Thus, computing an end-to-end path that meets the QoS constraints using BGP is not always possible, even if such a path exists. Furthermore, 
with BGP only one single path per destination can be propagated between two neighbor domains. These two limitations prevent BGP to deal with QoS routing.

The second type of algorithms are the exact ones. An algorithm is called exact if it can find a feasible end-to-end path, when such a path exists. As an exact algorithm, we choose the ID-MCP algorithm introduced by Bertrand et al [15]. This algorithm has the best success rate as it is exact. However, the complexity of executing ID-MCP in each domain corresponds to the complexity of the SAMCRA algorithm given by: $\mathcal{O}\left(\left(K_{\max } * N \log \left(K_{\max } * N\right)+\right.\right.$ $\left.\left.K_{\text {max }}^{2} m * E\right)\right)$, where $K_{\max }=\min \left(\exp (N-2) !, \frac{\prod_{i=1}^{m} c_{i}}{\max _{j} c_{j}}\right)$ [21]. This complexity is very high compared with that of BGP and HID-MCP algorithms.

We define the global success rate $(G S R)$ of the algorithms by the ratio of the number of requests for which a feasible path is found to the total number of requests. For the inter-domain crankback based HID-MCP, we choose the parameter hops $=q-1$. Thus, the crankback signaling will be sent to the destination domain $D_{1}$. We select this value for hops in order to study the performance of HID-MCP in the two extremity cases: intra-domain crankback and inter-domain crankback starting from the destination domain. For the other values of hops, the GSR of HID-MCP will be lower than that of HID$\mathrm{MCP}$ with hops $=q-1$ and higher than that of HID-MCP with hops $=0$.

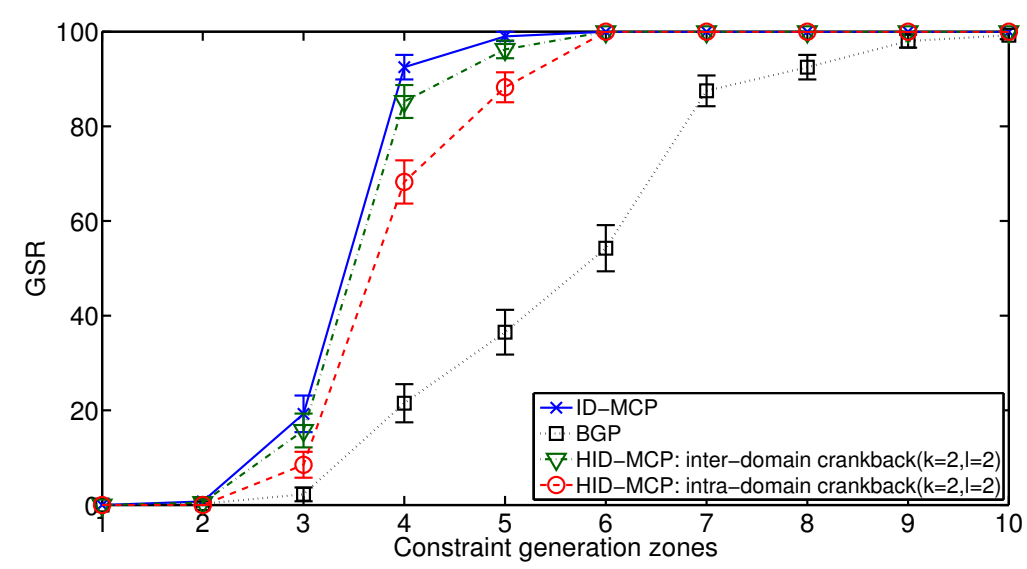

Fig. 10 Comparison of the global success rate of the algorithms in the Waxman's modelbased topology.

Fig. 10 illustrates the variation of the global success rate $(G S R)$ of HIDMCP with intra-domain crankback mechanism, HID-MCP with inter-domain crankback mechanism, the exact algorithm ID-MCP, and the BGP algorithm, in the Waxman's model-based topology. The BGP algorithm has the lowest success rate. This is due to the fact that only one path can be propagated from a domain to another neighbor domain using BGP, while with the other 
algorithms multiple paths can be propagated using the VSPH structure. The ID-MCP algorithm is an exact algorithm, while the others are heuristic ones. This is why the success rate of ID-MCP is the highest. We remark that the success rate of HID-MCP with inter-domain crankback when $l=2$ and $k=2$ is very close to the success rate of the ID-MCP. As explained in section $4, k$ is a parameter of TAMCRA and $l$ is the maximum number of paths selected from the VSPH for executing the TAMCRA computation. Of course, the higher $l$ and $k$, the closer the success rate to the optimal solution given by ID-MCP. Even so, with low values of $l$ and $k(l=2$ and $k=2)$, the success rate of the HID-MCP with inter-domain crankback is close to that of ID-MCP. As expected, the success rate of HID-MCP with intra-domain crankback when $l=2$ and $k=2$ is lower than that of HID-MCP with inter-domain crankback with the same parameters, especially in the middle constraint generation space. In fact, when the combination procedure fails, HID-MCP with inter-domain crankback executes the on-demand computation procedure starting from the destination domain, while HID-MCP with intra-domain crankback executes it only in the current domain. Consequently, the quality of the paths computed by the inter-domain crankback approach in each domain is better than the ones computed by the intra-domain crankback approach. Thus, the probability that a feasible path is found using HID-MCP with inter-domain crankback is higher. However, its computational complexity is high compared to HID-MCP with intra-domain crankback, but remains acceptable compared to ID-MCP.

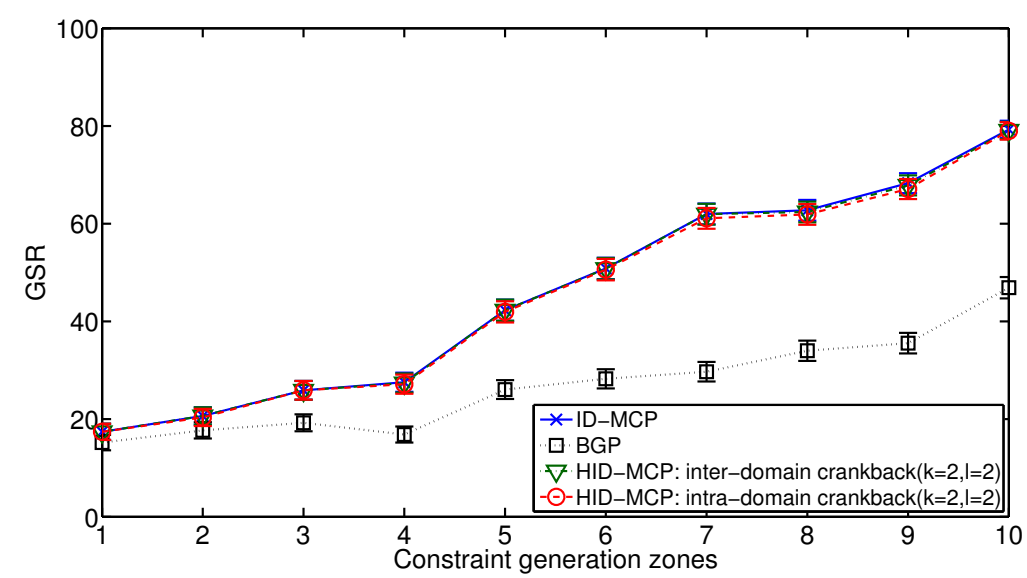

Fig. 11 Comparison of the global success rate of the algorithms in the SYM-CORE topology.

Finally, we evaluate the success rate of algorithms in the SYM-CORE topology. In Fig. 11, we see that the inter-domain crankback based HID-MCP, the intra-domain crankback based HID-MCP, and the ID-MCP algorithm have almost the same success rate, while BGP has the lowest success rate. The differ- 
ence of success rate between HID-MCP and BGP equals $30 \%$ in the constraint generation zone $Z_{10}$. The fundamental result deduced from these figures is that HID-MCP has a global success rate very high compared to that of BGP and very close to that of the exact algorithm.

\section{Conclusion}

In this paper, we studied the inter-domain QoS routing problem. We proposed a novel inter-domain QoS routing algorithm based on a hybrid computation scheme, named HID-MCP. Our algorithm relies on the PCE architecture. This architecture allows end-to-end path computation subject to multiple QoS constraints, while preserving the confidentiality clauses and the autonomy of the domains. We introduced two different mechanisms for improving the success rate of the HID-MCP algorithm. The first mechanism performs local improvement using an intra-domain crankback, while the second one executes a global improvement using an inter-domain crankback.

The simulation results showed that HID-MCP, with both of the aforementioned approaches, has a success rate very close to the optimal solution. Moreover, HID-MCP has a success rate very high compared with that of BGP and the gap is up to $30 \%$ in the realistic network SYMCORE. We also compared the average computational time of the path combination and the on-demand computation. This comparison study showed us the advantage of using precomputation in reducing the computational time of our proposed algorithm.

As future prospects of this work, the stability of the pre-computed paths should be analyzed when they deal with dynamic changes of link state. This allows inferring the validity of the pre-computed paths after an eventual change of the network conditions.

\section{References}

1. M. Yannuzzi, X. Masip-Bruin, S. Sanchez, J. Domingo-Pascual, A. Oreda, and A. Sprintson, On the challenges of establishing disjoint QoS IP/MPLS paths across multiple domains, IEEE Communications Magazine, vol. 44, no. 12, pp. 60-66, 2006.

2. S. Uludag, K. Lui, K. Nahrstedt, G. Brewster, Analysis of topology aggregation techniques for QoS routing, ACM Computing Surveys (CSUR), vol. 39, no. 3, paper 7, 2007.

3. A. Farrel, J.P. Vasseur, J. Ash, Path Computation Element (PCE)-Based Architecture, IETF RFC 4655, 2006.

4. J. Ash, and J. Le Roux, A path computation element (PCE) communication protocol generic requirements, IETF RFC 465\%, 2006.

5. P. Torab et al, On cooperative inter-domain path computation, In the Eleventh IEEE Symposium on Computers and Communications (IEEE ISCC), Sardinia, Italy, 2006.

6. J. P. Vasseur, et al, A Backward-Recursive PCE-Based Computation (BRPC) Procedure to Compute Shortest Constrained Inter-Domain Traffic Engineering Label Switched Paths, IETF RFC 5441, April 2009.

7. G. Geleji, H. Perros, Y. Xin, and T. Beyenne, A Performance Analysis of InterDomain QoS Routing Schemes Based on Path Computation Elements, HONET, pp. 146-152, 2008. 
8. A. Frikha, S. Lahoud, and B. Cousin, Hybrid Inter-Domain QoS Routing With Crankback mechanisms, In the 11th International Conference on Next Generation Wired/Wireless Advanced Networking (NEW2AN), St. Petersburg, Russia, 2011.

9. A. Orda, and A. Sprintson, QoS routing: the precomputation perspective,IEEE INFOCOM, vol. 1, pp. 128-136, 2000.

10. A. Orda, and A. Sprintson, Precomputation Schemes for QoS Routing, IEEE/ACM Transactions on Networking, vol. 11, no. 4, 2003.

11. Z. Ma, P. Zhang, R. Kantola, Influence of Link State Updating on the Performance and Cost of QoS Routing in an Intranet, IEEE Workshop on High Performance Switching and Routing (HPSR), Dallas, Texas USA, 2001.

12. Z. Wang, and J. Crowcroft, Quality-of-Service Routing for Supporting Multimedia Applications, IEEE Journal on Selected Areas in Communications, vol. 14, no. 7, pp. 1228$1234,1996$.

13. T. Knoll, BGP Extended Community Attribute for QoS Marking, draft-knoll-idr-qosattribute-02, work in progress, IETF, 2009.

14. D. Griffin, J. Spencer, J. Griem, M. Boucadair, P. Morand, M. Howarth, N. Wang, G. Pavlou, A. Asgari, and P. Georgatsos, Interdomain routing through QoS-class planes, IEEE Communication Magazine, vol. 45, no. 2, pp. 88-95, 2007.

15. G. Bertrand, S. Lahoud, G. Texier, and M. Molnar, A Distributed Exact Solution to Compute Inter-domain Multi-constrained Paths, The Internet of the Future, Lecture Notes in Computer Science, vol. 5733, pp. 21-30, 2009.

16. P. Van Mieghem and F. A Kuipers, Concepts of exact QoS routing algorithms, IEEE/ACM Transactions on Networking, vol. 12, no. 5, pp. 851-864, 2004.

17. A. Frikha, S. Lahoud, Pre-computation Based Heuristic for Inter-Domain QoS Routing, In Fourth IEEE International Conference on Advanced Networks and Telecommunication Systems (IEEE ANTS), Mumbai, India, 2010.

18. A. Frikha, S. Lahoud, Performance Evaluation of Pre-computation Algorithms for InterDomain QoS Routing, In the 18th International Conference on Telecommunications, Ayia Napa, Cyprus, 2011.

19. M. Esmaeili, F. Xu, M. Peng, N. Ghani, A. Gumaste and J. Finochietto, Enhanced Crankback Signaling for Multi-Domain IP/MPLS Networks, Computer Communications, vol. 33, no. 18, pp. 2215-2223, 2010.

20. M. Molnar, Hierarchies for Constrained Partial Spanning Problems in Graphs, IRISA, Tech. Rep. 1900, 2008.

21. P. Van Mieghem, H. De Neve, and F. A. Kuipers, Hop-by-hop quality of service routing, Computer Networks, 37, 407-423, 2001.

22. K. I. Calvert, M.B. Doar, and E.W. Zegura, Modelling Internet Topology, IEEE Communications Magazine, vol. 35, no. 6, pp. 160-163, 1997.

23. J. Guichard, F. Le Faucheur, and J.P. Vasseur, Definitive MPLS Network Designs, Cisco Press, 2005. 